Proceedings

\title{
Powering E-Textiles Using a Single Thread Radio Frequency Energy Harvesting Rectenna ${ }^{\dagger}$
}

\author{
Mahmoud Wagih *, Alex S. Weddell and Steve Beeby
}

\author{
School of Electronics and Computer Science, University of Southampton, Southampton SO17 1BJ, UK; \\ asw@ecs.soton.ac.uk (A.S.W.); spb@ecs.soton.ac.uk (S.B.) \\ * Correspondence: mahm1g15@ecs.soton.ac.uk; Tel: +44-2380-593-234 \\ + Presented at the International Conference on the Challenges, Opportunities, Innovations and Applications \\ in Electronic Textiles (E-Textiles 2020), Virtual venue, UK, 4 November 2020.
}

\begin{abstract}
Radio frequency energy harvesting (RFEH) and wireless power transfer (WPT) are increasingly seen as a method of enabling sustainable computing, as opposed to mechanical or solar EH WPT does not require special materials or resonators and can be implemented using low-cost conductors and standard semiconductor devices. This work revisits the simplest antenna design, the wire monopole to demonstrate the lowest-footprint, lowest-cost rectifying antenna (rectenna) based on a single Schottky diode. The antenna is fabricated using a single Litz-wire silk-coated thread, embroidered into a standard textile substrate. The rectifier is fabricated on a compact lowcost flexible printed circuit board (PCB) using ultra-thin polyimide copper laminates to accommodate low-footprint surface mount components. The antenna maintains its bandwidth across the $868 / 915 \mathrm{MHz}$ license-free band on- and off-body with only $-4.7 \mathrm{~dB}$ degradation in total efficiency in human proximity. The rectenna achieves up to $55 \%$ RF to DC efficiency with $1.8 \mathrm{~V}$ DC output, at 1 $\mathrm{mW}$ of RF power, demonstrating its suitability as a power-supply unit for ultra-low power e-textile nodes.
\end{abstract}

Keywords: antenna; e-textiles; energy harvesting; rectenna; wireless power transfer

Citation: Wagih, M.; Weddell, A.S.; Beeby, S. Powering E-Textiles Using a Single Thread Radio Frequency Energy Harvesting Rectenna. Proceedings 2021, 68, 16. https:// doi.org/10.3390/proceedings 2021068016

Published: 25 January 2021

Publisher's Note: MDPI stays neutral with regard to jurisdictional claims in published maps and institutional affiliations.

Copyright: $\left(C^{2} 2021\right.$ by the authors. Licensee MDPI, Basel, Switzerland. This article is an open access article distributed under the terms and conditions of the Creative Commons Attribution (CC BY) license (http://creativecommons.org/licenses/by/4.0/).

\section{Introduction}

Radio frequency (RF) energy harvesting (EH) is a potential method of powering electronic-textiles sustainably, enabling battery-free operation. RF energy can either be scavenged from ambient wireless networks such as cellular or Wi-Fi, or directly transmitted to the e-textile device using a dedicated transmitter such as a RF-ID interrogator [1]. A rectifying antenna (rectenna), formed of a radiating element whose impedance is matched to a rectifier is required to convert the available RF power to DC power for storage and regulation by a power management circuit. The rectifier is commonly based on Schottky diodes of different topologies depending on the application and target power levels, with the most popular topologies being the single-series, aimed at maximizing the RF-DC efficiency for $\mu \mathrm{W}$-RFEH, or a voltage multiplier and charge-pump, aimed at improving the voltage sensitivity of the harvester at $\mathrm{mW}$ and sub-mW RFEH and WPT applications; a review of the performance of different rectifier is available in [1].

The sub-1 GHz Industrial Scientific and Medical (ISM)-band (Europe-868/US-915 $\mathrm{MHz}$ ) represent an attractive channel for RFEH and WPT for autonomous body area networks, due to the lower free space propagation losses and the potential to achieve higher rectification efficiency than higher frequency microwave and mmWave bands. Thus, Multiple low-power transceivers and energy harvesters have been presented for the $915 \mathrm{MHz}$ band [1]. The only trade-off associated with RFEH at sub-1 GHz bands is the antenna size due to the wavelength. However, in e-textiles the area of the device is not limited and multiple rectennas could co-exist on the same garment. 
Although harvesting on textiles has been considered at different frequencies form 2.4 $\mathrm{GHz}$ to mmWave bands [2,3], the main focus of the work has been on achieving highefficiency harvesting on the expense of ease of integration in any textile without requiring low-loss materials or thick fabrics. For example, reported microstrip patch antennas have been limited to thick felt fabric to ensure high radiation efficiency. Moreover, the rectifier has been integrated on a rigid low-loss RF substrate (RT/Duroid) which increase the design complexity and cost [2].

This work presents a fully-flexible textile rectenna based on a single-thread microstrip monopole antenna with a miniaturised ground plane. The rectifier, designed on an ultra-thin flexible polyimide PCB, achieves a high RF-DC efficiency of up to $60 \%$ at 1 $\mathrm{mW}$ of RF power level with $1.8 \mathrm{~V}$ when connected to a $6.8 \mathrm{kOhm}$ load. This paper discusses the thread-monopole antenna design and fabrication, the performance of the antenna on and off-body, as well as the textile rectifier's performance for EH applications.

\section{Embroidered Textile Monopole Antenna}

The simplest antenna design is a quarter-wavelength wire perpendicular to an infinite ground plane. However, on a planar textile surface it is impossible to realise a freestanding antenna. Moreover, a large ground plane will affect the user's comfort due to the added weight and reduced flexibility of the fabric. A planar microstrip monopole antenna has been designed and simulated in CST Microwave Studio, a full-wave 3D electromagnetic simulation package. The ground plane size has been minimised with the length of the monopole tuned empirically based on the simulation results to achieve resonance at $915 \mathrm{MHz}$. Figure 1 shows the layout of the designed thread monopole antenna. The 20$\mathrm{mm}$ wide has been designed of 50-mm length to improve and stabilise the $50-\mathrm{Ohm}$ matching of the antenna.

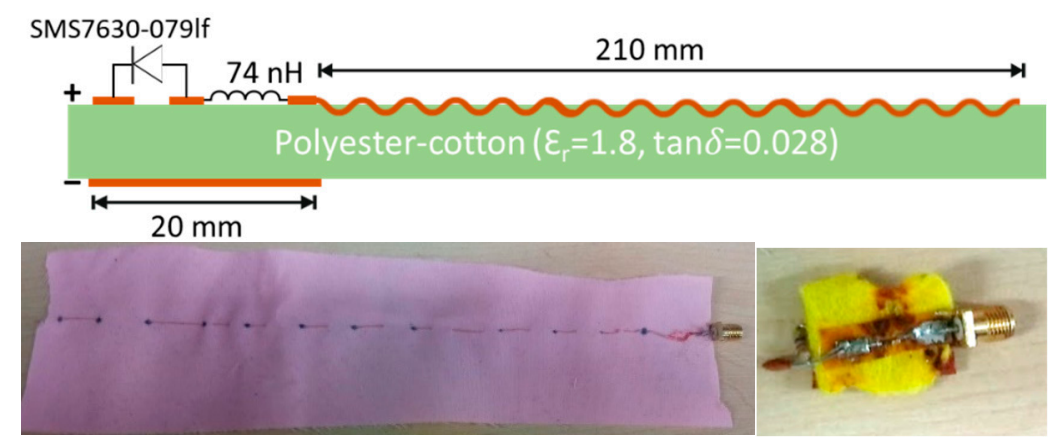

Figure 1. (top)The fabricated rectenna layout (not to-scale). (bottom) Photograph of the sewn antenna (left) and rectifier (right), on a poly-cotton and a felt substrates respectively.

The proposed antenna has been fabricated on a polyester-cotton substrate $\left(\varepsilon_{\mathrm{r}}=1.8\right.$, $\tan \delta=0.028$ [2]). Conductive silk-coated Litz copper wires, of $40 \mu \mathrm{m}$ thickness, have been stitched to the polyester-cotton fabric to form the antenna's radiator. Stitched or embroidered Litz wires have been chosen due to their higher conductivity compared to printed silver-paste conductive inks. Figure 2 shows an SEM cross-section of the conductive threads. The Litz wire has been soldered to the $50 \mathrm{Ohm}$ microstrip line connected to the rectifier. The microstrip rectifier and matching network have been fabricated on a $25 \mu \mathrm{m}$ substrate. 


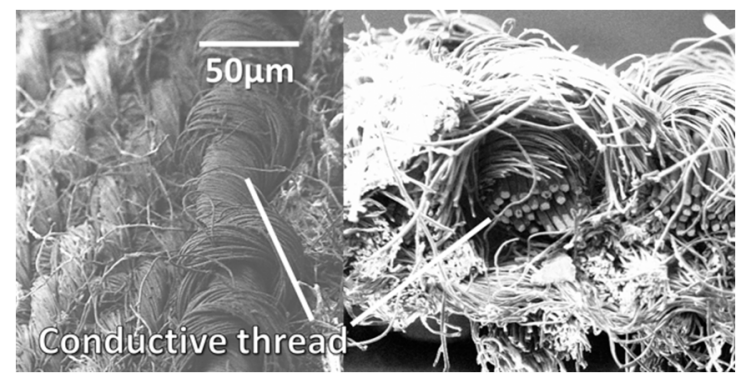

Figure 2. Scanning electron microscope (SEM) image of the surface (left) and cross-section (right), showing the conductive Litz wires, of the antenna.

The fabricated antenna's bandwidth has been measured using a Rohde \& Schwarz ZVB4 (Germany) vector network analyzer (VNA). Figure 3 shows the measured return loss of the antenna, showing its bandwidth covering the $915 \mathrm{MHz}$ ISM-band. The antenna's radiation characteristics have been obtained from CST 3D field monitors in simulation as well as measured experimentally. The forward transmission between two identical textile antennas has been measured with the path losses estimated using the Friis free space propagation model. Table 1 shows the simulated and measured antenna characteristics, demonstrating higher radiation efficiency than textile antennas, such as [2], due to the lower dielectric losses associated with a monopole antenna compared to a microstrip patch, with minimal impact of the substrate's thickness and dissipation factor.

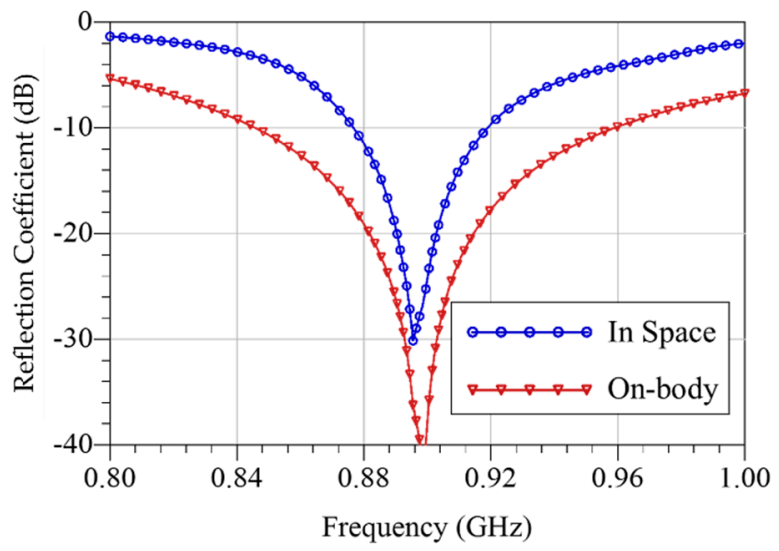

Figure 3. Measured return loss of the single-thread monopole, with a large ground plane, showing a $4.6 \%$ fractional impedance bandwidth.

Table 1. Single-thread monopole antenna characteristics.

\begin{tabular}{ccc}
\hline & In Space & On-Body \\
\hline Bandwidth $(\mathrm{MHz})$ & 42.2 & 114.6 \\
\hline Fractional Bandwidth & $4.6 \%$ & $12.7 \%$ \\
\hline Simulated Gain (dBi) & 2.1 & - \\
Measured Realised Gain (dBi) & 1.9 & -2.8 \\
\hline Simulated Radiation Efficiency & $35 \%$ & $0.58 \%$ \\
\hline Radiation Efficiency & $89 \%$ & - \\
\hline
\end{tabular}

To seamlessly integrate the antenna in textiles, the ground plane needs to be minimized to the minimum area which fits the rectifier's components. However, the $50 \mathrm{Ohm}$ matching of the monopole is expected to be affected by the size reduction. Figure 4 shows the measured return loss and gain of the stitched monopole with different ground-plane sizes. While it is observed that the impedance matching quality reduces with ground planes smaller than $25 \mathrm{~cm}^{2}$, the impact on the realized gain has been less than $-2 \mathrm{~dB}$ demonstrating the suitability of a textile monopole of $2 \mathrm{~cm}^{2}$ ground plane. 

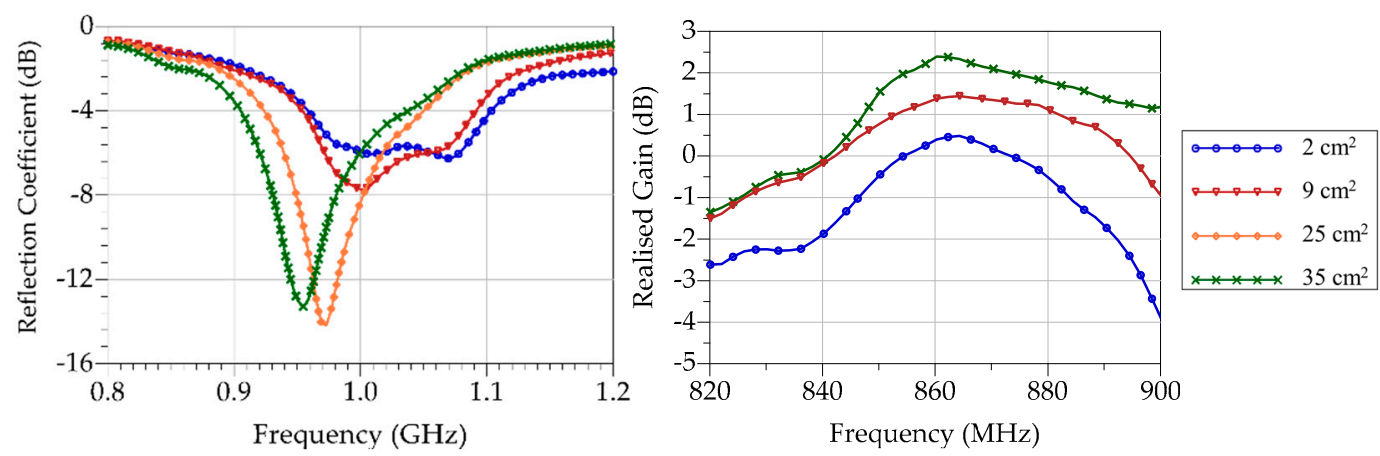

Figure 4. Effect of varying the ground plane's size on a $0.67 \lambda$-length monopole's measured bandwidth (left) and realised gain (right).

\section{Textile Rectifier}

The proposed single series rectifier, shown in Figure 1, has been designed on a 50 Ohm microstrip line soldered to the antenna's output. A 1st order L-matching network has been designed to match the antenna's 50 Ohm impedance to the diode's complex impedance at $915 \mathrm{MHz}$, obtained using harmonic balance simulation. $50 \mathrm{Ohm}$ matching networks simplify the rectifier characterization process using standard lab instruments, and enable the use of standard antenna designs, as opposed to antenna-rectifier co-design [4]. The diode is a Skyworks SMS7630-079 Schottky. The power conversion efficiency (PCE) of the rectifier has been tested for different RF power levels and load impedances, Figure 5 shows the rectifier's PCE for varying RF power levels and load impedances.
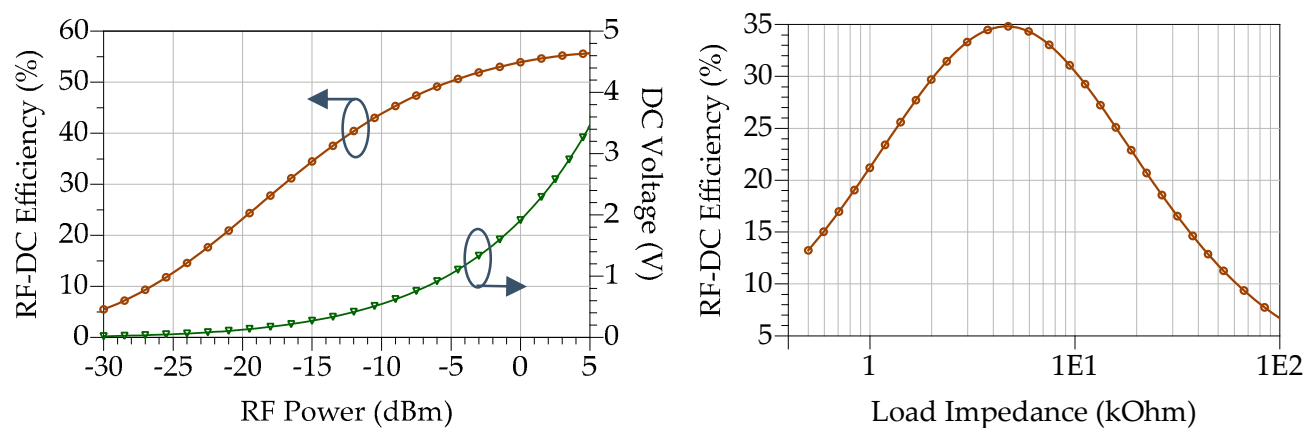

Figure 5. RF-DC Power conversion efficiency of the textile rectifier for different RF power levels (left) and load impedances (right).

While the observed PCE is lower than the ideal PCE achievable by a matched SMS7630-079 diode as in [1,2], the losses are attributed to the inductor having a series resistance of approximately $10 \mathrm{Ohms}$, calculated using the circuits model in ADS using harmonic balance. This could be overcome by utilising a high-Q inductor, or a distributed matching network on a less-lossy substrate.

\section{Conclusions}

This paper presented a low-cost fully-textile rectenna demonstrating the potential of RF wireless power acting as a seamlessly-integrated power supply for e-textiles. A simple antenna design and a fabric-independent rectifier have been presented demonstrating high RF-DC efficiency and low optimal output load impedance for ease of integration with power management circuitry. Future work includes investigation of a large rectenna array with DC-combining for high-RF-power reception, in addition to methods of reducing mutual-coupling between multiple on-body rectennas, as well as utilizing RF power to power a wearable all-textile wireless sensor node. 
Funding: This work was supported by the European Commission under H2020-EU.1.4.1.2. Grant number: 730957

The study was conducted according to the guidelines of the Declaration of Helsinki, and approved by the Ethics and Research Governance Committee of the University of Southampton (submission $54082,02 / 01 / 2020)$

Informed Consent Statement: Informed consent was obtained from all subjects involved in this study.

Data Availability Statement: Datasets supporting this paper are available from the corresponding author upon request.

\section{References}

1. Valenta, C.R.; Durgin, G.D. Harvesting Wireless Power: Survey of Energy-Harvester Conversion Efficiency in Far-Field, Wireless Power Transfer Systems. IEEE Microw. Mag. 2014, 15, 108-120.

2. Adami, S.E.; Proynov, P.; Hilton, G.S.; Yang, G.; Zhang, C.; Zhu, D.; Li, Y.; Beeby, S.P.; Craddock, I.J.; Stark, B.H. A Flexible 2.45$\mathrm{GHz}$ Power Harvesting Wristband with Net System Output From $-24.3 \mathrm{dBm}$ of RF Power. IEEE Trans. Microw. Theory Tech. 2018, 66, 380-395.

3. Wagih, M.; Hilton, G.S.; Weddell, A.S.; Beeby, S. Broadband Millimeter-Wave Textile-Based Flexible Rectenna for Wearable Energy Harvesting. IEEE Trans. Microw. Theory Tech. 2020, 68, 4960-4972.

4. Wagih, M.; Weddell, A.S.; Beeby, S. Rectennas for Radio-Frequency Energy Harvesting and Wireless Power Transfer: A Review of Antenna Design [Antenna Applications Corner]. IEEE Antennas Propag. Mag. 2020, 62, 95-107. 